Artículos científicos

\title{
Modelo de gestión de capital humano por competencias para universidades públicas en México
}

Human Capital Management Model by Competencies for Public Universities in México

Modelo de gestão de capital humano por competências para universidades públicas no México

Lozano Rosales Raymundo Universidad Politécnica de Tulancingo, México

ray_mundo111@yahoo.com.mx https://orcid.org/0000-0001-6856-4265

María de Lourdes Amador Martínez Tecnológico Nacional de México, Instituto Tecnológico Superior del Oriente del Estado de Hidalgo, México lamador@itesa.edu.mx https://orcid.org/0000-0001-8963-6264

Mariza Raluy Herrero Universidad Politécnica de Tulancingo, México mariza.raluy@upt.edu.mx https://orcid.org/0000-0001-8516-1337 


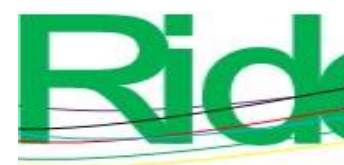

Revista Iberoamericana para la Investigación y el Desarrollo Educativo ISSN $2007-7467$

\title{
Resumen
}

El capital humano es pieza indispensable en cada organización, por lo que la gestión de este debe ser punto de partida en cualquier entidad. El objetivo de esta investigación fue generar un modelo de gestión de capital humano para universidades públicas en México cuyo modelo educativo se sustente en la promoción de competencias. El estudio se realizó bajo un enfoque cualitativo in situ y tuvo un corte transversal. Se utilizó como base el paradigma de investigación de la teoría fundamentada (grounded theory). La recolección de datos se llevó a cabo mediante entrevistas y la técnica de observación. El modelo que se diseñó se encuentra constituido por tres etapas: 1) reclutamiento y selección docente por competencias, 2) contratación y 3) desarrollo. Se trata de una propuesta de modelo cíclico, pero no cerrado, perfectible en sus conceptos y abierto a mejoras tanto administrativas como de fundamentos legales.

Palabras clave: competencias del docente, enseñanza pública, gestión de personal, modelo educacional, universidad.

\begin{abstract}
Human capital is an indispensable piece in every organization, so its management must be the starting point in any entity. The objective of this research was to generate a human capital management model for public universities in Mexico whose educational model is based on the promotion of skills. The study was carried out under a qualitative approach in situ and had a cross section. The grounded theory research paradigm was used as a basis. Data collection was carried out through interviews and the observation technique. The model that was designed is made up of three stages: 1) teacher recruitment and selection by competencies, 2) hiring and 3) development. It is a proposal for a cyclical but not closed model, perfectible in its concepts and open to improvements, both administrative and legal. Keywords: teacher competencies, public education, personnel management, educational model, university.
\end{abstract}




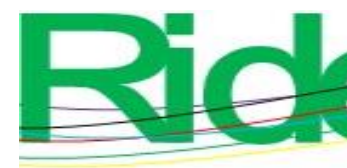

Revista Iberoamericana para la Investigación y el Desarrollo Educativo ISSN 2007 - 7467

\section{Resumo}

O capital humano é uma peça indispensável em qualquer organização, portanto sua gestão deve ser o ponto de partida em qualquer entidade. O objetivo desta pesquisa foi gerar um modelo de gestão do capital humano para universidades públicas no México, cujo modelo educacional é baseado na promoção de competências. O estudo foi realizado sob uma abordagem qualitativa in loco e teve um corte transversal. O paradigma de pesquisa da teoria fundamentada foi usado como base. A coleta de dados foi realizada por meio de entrevista e da técnica de observação. O modelo desenhado é composto por três etapas: 1) recrutamento e seleção de professores por competências, 2) contratação e 3) desenvolvimento. É uma proposta de modelo cíclico, mas não fechado, aperfeiçoável em seus conceitos e aberto a melhorias, tanto administrativas quanto jurídicas.

Palavras-chave: competências docentes, educação pública, gestão de pessoas, modelo educacional, universidade.

Fecha Recepción: Julio 2021

Fecha Aceptación: Diciembre 2021

\section{Introducción}

Una organización se compone de dos personas o más que forman un sistema estructurado diseñado para lograr metas y objetivos en común. Uno de los fines de cualquier empresa es ser competitiva en un mercado global. Para lograr lo anterior, es necesario asegurar la calidad de sus productos o servicios. De ahí que el factor humano sea indispensable para un buen funcionamiento organizacional. En efecto, la mayoría de las empresas cuentan con procesos propios de capacitación, reclutamiento y selección de personal cuyo propósito es adquirir el talento humano idóneo para cada puesto, calificarlo para las tareas, proyectos y objetivos asignados según la actividad a la que se dedique.

Actualmente, en la sociedad del conocimiento, los procesos relacionados con el capital humano han adquirido un nuevo cariz de importancia debido a que de la eficiencia de estos depende en gran medida el logro de los objetivos de la organización. Es por ello por lo que generar un modelo de gestión de capital humano como propuesta para las universidades públicas está íntimamente relacionado con la capacidad de las empresas de alcanzar sus objetivos. Se trata de entablar un esfuerzo coordinado entre los responsables dirigido hacia la mejora continua de la gestión de personal: la conformación de un modelo de gestión de 


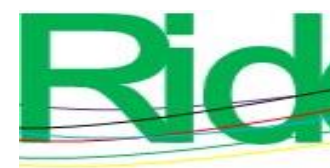

Revista Iberoamericana para la Investigación y el Desarrollo Educativo ISSN 2007 - 7467

capital humano docente dependerá de las necesidades organizacionales, así como de la aportación de la gestión por competencias de los organismos gubernamentales correspondientes.

Una vez realizado el estudio, el análisis y la sistematización de datos, y siguiendo una metodología cualitativa, el presente trabajo propone un modelo de gestión de capital humano docente donde se incluyen las competencias básicas y específicas que debe cumplir un candidato, así como las competencias que deberán desarrollar los docentes y las personas que integren el departamento de recursos humanos como parte de un modelo conductual de competencias.

\section{Revisión de la literatura}

Según Giere (2004), un aspecto relevante de los modelos científicos es su capacidad para representar aspectos del mundo: "Los científicos usan modelos para representar aspectos del mundo con diversos propósitos. Desde este punto de vista, los modelos son instrumentos primarios de representación en las ciencias" (p. 747). Esta capacidad de los modelos reside en que "están diseñados para que sus elementos puedan identificarse con características del mundo real" (Giere, 2004, p. 747).

Bailer-Jones (2013), por su parte, caracteriza a los modelos científicos como descripciones interpretativas de fenómenos que facilitan el entendimiento de estos, de los fenómenos. Así, identifica que los modelos no solo deben encajar con los datos empíricos disponibles, sino también deben dar lugar a predicciones y, de esta manera, ser contrastables.

Mientras que Suárez (2010) indica que los modelos científicos no son entidades mentales ni lingüísticas; pueden ser entidades concretas, como una maqueta o un prototipo, o entidades abstractas, como una estructura conjuntista o un sistema de ecuaciones, pero, en cualquier caso, no tienen un carácter mental o lingüístico.

En el mismo tenor, Adúriz e Izquierdo (2009) indican que el modelo es la relación entre la teoría y el mundo o la relación específica entre los modelos de la teoría y el mundo. La relación de similitud de los modelos se fundamenta a partir de la conexión entre sí y la realidad. También señalan que el modelo funge el papel de mediador entre la teoría y los datos empíricos, o entre la teoría y el mundo real.

Finalmente, Acevedo, García, Aragón y Oliva (2017) señalan que un modelo científico es como una representación de un objetivo (el referente) y que los referentes 

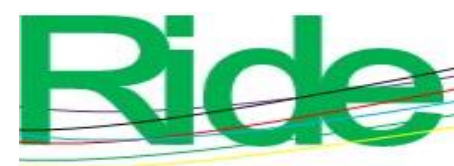

Revista Iberoamericana para la Investigación y el Desarrollo Educativo ISSN 2007 - 7467

representados por los modelos pueden ser diversas entidades, tales como objetos, fenómenos, procesos, ideas o sistemas. Un modelo científico también es una herramienta para conectar una teoría científica con un fenómeno, porque ayuda al desarrollo de la teoría desde los datos y la vincula con el mundo natural.

A continuación, en la figura 1, se retoman algunos de estos elementos centrales para poder entender lo que es un modelo científico. Cabe advertir que este tiene características específicas de acuerdo con el área de estudio.

Figura 1. Elementos conceptuales de un modelo científico

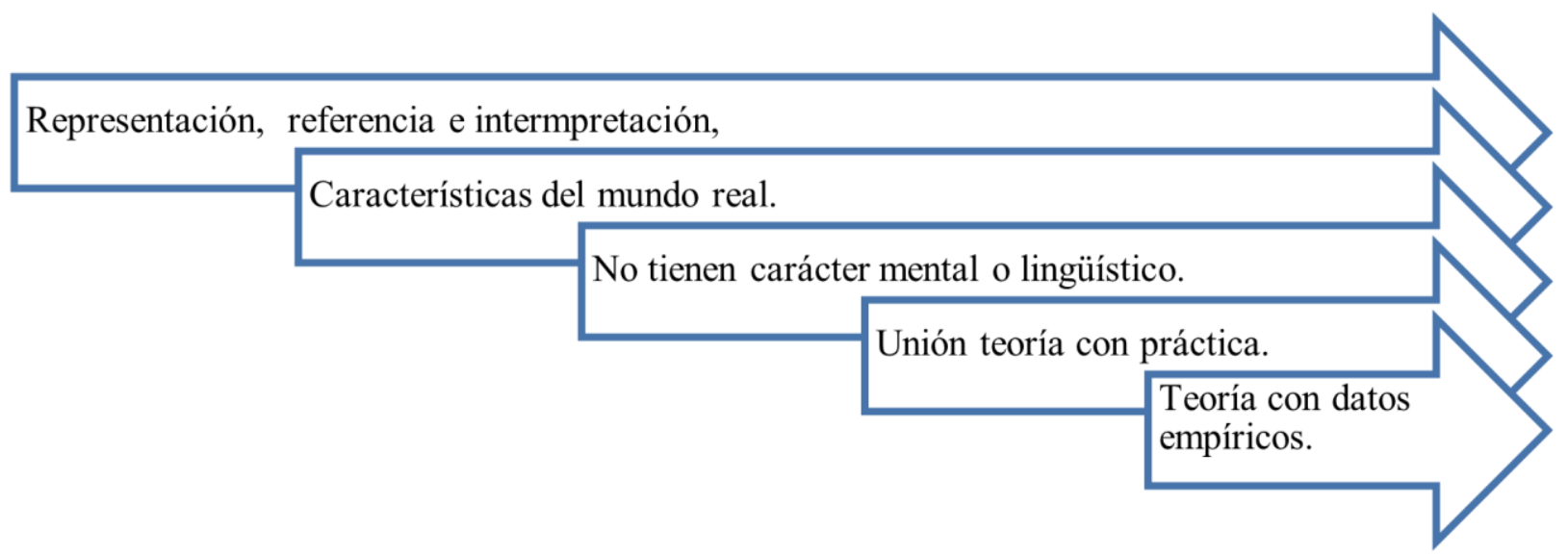

Fuente: Elaboración propia

Una vez abordado el concepto de modelo, es necesario tratar la gestión de capital humano. Cuesta y Valencia (2018) indican que la gestión de capital humano incluye todas las decisiones y acciones directivas que afectan la relación entre los empleados, la organización y el trabajo que se va desarrollando. Asimismo, Rodríguez (2015) considera que la gestión de recursos humanos consiste en la planeación, organización, desarrollo y coordinación, así como también en el control de técnicas capaces de promover el desempeño eficiente del personal. En esta concepción, la organización representa el medio que permite a las personas que colaboran en ella alcanzar los objetivos individuales relacionados directa o indirectamente con el trabajo.

Hellriegel (2009) propone un enfoque basado en competencias y combina los principios de la administración con lo que él considera como competencias centrales, a saber: autoadministración, acción estratégica, multicultura, trabajo en equipo, planeación, administración, y comunicación. Además, paralelamente, presenta las etapas de la administración. Así, Hellriegel (2009) hace un análisis que para la presente investigación es 


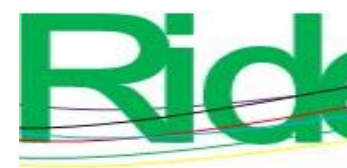

Revista Iberoamericana para la Investigación y el Desarrollo Educativo ISSN 2007 - 7467

importante dado que combina las fases de la administración desde una óptica de las competencias.

Las entrevistas por competencias son, para el mismo autor, un factor relevante. Las entrevistas pueden ser de utilidad para obtener mejores resultados, sobre todo si se realiza con un formato donde las competencias sean el centro de esta. Además, fundamenta que ofrecer capacitación a las personas que dan empleo ayuda a los empleados a superar restricciones laborales e incrementar su capacidad productiva, puesto que fomenta las competencias necesarias para desarrollar el trabajo y su entorno de vida.

Alles (2017) por su parte, identifica los elementos de un seguimiento integral para cubrir una vacante: inicia con la determinación de reclutar y termina con el proceso de promoción de la persona. Las siguientes fases forman parte de este proceso: 1) gestión por competencias, 2) selección por competencias, 3) planificación, 4) definición del perfil, 5) atracción y reclutamiento, 6) primera selección, 7) entrevista por competencias, 8) comparación de candidatos, 9) evaluaciones específicas, 10) negociación y oferta, 11) incorporación, 12) control de gestión, 13) auditoría y 14) promociones internas.

De lo anterior vale la pena destacar la incursión de las competencias en las entrevistas y en el proceso de promoción, además de observar el perfil de la persona, dado que son elementos que permiten formar una visión más integral y que otorgan mayor cantidad de herramientas al proceso de gestión de capital humano en las organizaciones.

Otro modelo de reclutamiento y selección de talento humano por competencias es el de Restrepo, Landino y Orozco (2008), quienes exponen una forma de elegir nuevos colaboradores con estándares suficientes para coadyuvar al desarrollo empresarial. Se trata este de un sistema de gestión humana que hace énfasis en la persona, sus capacidades y motivaciones como factores de éxito.

Las autoras identifican los siguientes elementos en el modelo desarrollado:

- Identificar las competencias.

- Reclutamiento.

- $\quad$ Proceso de selección de talento Humano.

- $\quad$ Fase de evaluación del proceso de selección.

Ahora bien, como parte de los trabajos que llevan la teoría a la práctica, Gómez y Mendoza (2013) presentan un modelo de gestión por competencias para la empresa Acmed, donde se destaca un manual de funciones y perfiles por competencias del personal. Y Saracho 


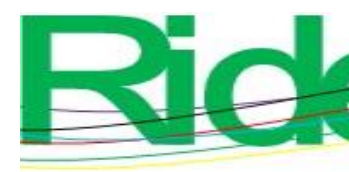

Revista Iberoamericana para la Investigación y el Desarrollo Educativo ISSN 2007 - 7467

(2005) traza una ruta que va desde la identificación de las competencias distintivas hasta los recursos necesarios para la puesta en marcha de un modelo.

Llama la atención la obra de Alles (2018), que propone un modelo de competencias donde se identifican las competencias cardinales, los niveles ejecutivos, los niveles intermedios y los niveles iniciales.

Es de hacer notar igualmente la propuesta de Tejada (2003), quien subraya el papel del aprendizaje en las competencias y sus subprocesos. Además, clasifica a las competencias desde la perspectiva de los procesos fundamentales de desarrollo gerencial. Esto resulta muy interesante, pues es un área de oportunidad de desarrollo de competencias y formación de directivos en las organizaciones.

Por su parte, Chiavenato (2020) plantea cuatro subsistemas: de alimentación, de aplicación, de mantenimiento y desarrollo, este último, como se verá más adelante, muy importante para el modelo propuesto en la presente investigación.

Finalmente, Cuesta (2005) plantea cuatro subsistemas: el flujo de recursos humanos, educación y desarrollo, sistemas de trabajo y compensación laboral. Posteriormente, presenta los aspectos fundamentales incluidos en cada uno de los elementos que constituyen su modelo: factores de situación, grupos de interés, políticas, recursos humanos y resultados.

El objetivo de la presente investigación fue proponer un modelo de gestión de capital humano docente dirigido a las universidades públicas en México que manejan un modelo educativo por competencias. Lo anterior con el fin de proponer mejoras en los procesos de gestión de personal docente.

\section{Supuesto de investigación}

Un modelo de gestión de capital humano para las universidades públicas que tienen como base educativa a las competencias puede lograr mejoras en los procesos de gestión de personal en los docentes de las universidades públicas.

\section{Materiales y métodos}

El uso de la metodología cualitativa fue elemental para conseguir el objetivo. Dada su orientación inductiva, en esta metodología no se busca probar hipótesis a priori. Además, permite contextualizar las vivencias de los maestros y personal administrativo, quienes, en 


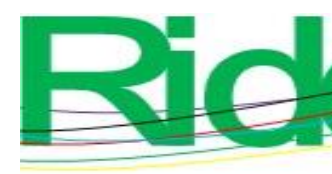

Revista Iberoamericana para la Investigación y el Desarrollo Educativo ISSN 2007 - 7467

su actuar diario en las universidades públicas, han vivido la transición de un modelo tradicional a la implementación del modelo por competencia. Esto resulta valioso, ya que son ellos quienes como informantes narrarán las experiencias vividas en su labor docente o administrativa diaria.

\section{Uso de la teoría fundamentada}

La presente investigación tiene como base el paradigma de la teoría fundamentada (grounded theory) de Glaser y Strauss (2017). La falta de una gestión de capital humano por competencias es una realidad en las instituciones públicas objeto de estudio que puede ser analizada mediante la narrativa de los informantes. En primer lugar, algunos docentes compartieron sus vivencias respecto de las dificultades que vivieron desde su reclutamiento hasta el instante en el que se enfrentaron al grupo de aprendizaje en un ambiente de aula de clases (virtual o presencial). En segundo lugar, se tuvo al personal administrativo, quienes, como informantes secundarios, expresaron oralmente y mediante documentos la forma de llevar a cabo la gestión de capital humano docente.

Se debe considerar que las hipótesis se lograron como producto de la metodología seleccionada y al término de la investigación, siempre con la finalidad de reunir información suficiente mediante entrevistas, las cuales permitieron saber a profundidad el estado que guarda la gestión de capital humano docente en el momento de desarrollo de la investigación. No está de más señalar, por último, que los informantes revisaron y consintieron los datos vertidos en las entrevistas.

\section{Reglas analíticas de la teoría fundamentada}

En la presente investigación no se pretendió comprobar hipótesis, sino que se generaron conceptos en el modelo presentado. Asimismo, se pretendió comprender y explicar el fenómeno de la gestión de capital humano por competencias en universidades públicas en México. La utilización de la teoría fundamentada se debió, además, porque se pretendió tener una abstracción generada por el actuar diario y su significación de los sujetos de investigación. En este sentido, se dio una abstracción del tiempo, espacio y sujetos de investigación.

Ahora bien, también se dio seguimiento a las reglas analíticas de la teoría fundamentada, siguiendo aquí a Raymond (2005), a saber: 


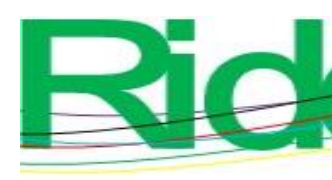

Revista Iberoamericana para la Investigación y el Desarrollo Educativo ISSN 2007 - 7467

- Definición del objeto de investigación: al estudiar los actos sociales de los docentes de las universidades públicas en México el objeto de investigación es el fenómeno social de la gestión de capital humano por competencias en las universidades públicas, esto considerado como una acción social susceptible de compararse de manera constante con otras situaciones similares y contrastantes, considerando que puede haber variaciones desde el inicio hasta el término del estudio.

- Sensibilidad teórica: es importante señalar que se buscó evitar las percepciones preconcebidas sobre la gestión de capital humano por competencias y se aludió a la capacidad creativa dada la experiencia profesional y personal de los investigadores de este trabajo.

Durante la investigación se requirió que los investigadores pensaran en términos teóricos y que los investigadores intervinieran constantemente en las operaciones de recopilación y análisis, en lugar de elaborar hipótesis hasta que los datos estén analizados.

- La selección de la situación o grupo bajo estudio: se llevaron a cabo observaciones y entrevistas para recopilar la información. También se buscó involucrarse en el actuar diario de los sujetos de investigación en los contextos de estudio.

- $\quad$ El muestreo teórico: quedó estrechamente vinculado con el análisis de la investigación de tal manera que las situaciones bajo estudio, los grupos y sujetos de investigación fueron seleccionados con base en las categorías previamente definidas, determinadas a su vez por la pregunta de investigación. Por lo mismo, resultó muy difícil fijarlo de antemano, pues se fue elaborando en etapas subsecuentes y desarrollándose a lo largo de la investigación.

- La elaboración de categorías conceptuales: tomando en consideración el principio de saturación, no se pretendió caracterizar datos, sino encontrar lo que significa la gestión de capital humano por competencias. De esta manera, los conceptos que iban surgiendo de un mismo universo se fueron agrupando en una categoría conceptual; aun con todo, no se buscó tanto una descripción minuciosa de los incidentes, los cuales van variando a lo largo de la investigación, sino que los conceptos se fueron afinando a partir de las comparaciones constantes del fenómeno.

- $\quad$ El proceso de codificación: la comparación fue la principal herramienta de análisis de la teoría fundamentada en la presente investigación; mostró similitudes y diferencias de los datos con el objetivo de identificar sus características y la relación entre 


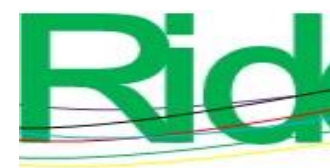

Revista Iberoamericana para la Investigación y el Desarrollo Educativo ISSN $2007-7467$

ellos. La codificación fue abierta, pues se codificaron todos los incidentes, sin embargo, a lo largo de la investigación se convirtió más selectiva; también fue acompañada de una reflexión teórica. La coherencia teórica no significó una inmovilidad de las categorías, por el contrario, se fueron adecuando con base en la generalidad de los datos.

Es importante recalcar que se dio importancia a los códigos y su interrelación; aquellos que quedaban fuera se omitieron.

- Las cualidades de la teoría substantiva: la aplicación de la teoría fundamentada en el modelo de gestión de capital humano por competencias permite integrar una serie de conceptos para generar una teoría flexible y la oportunidad de estudiar el fenómeno de la gestión de capital humano a profundidad.

\section{Variables}

No se consideraron variables discretas dada la metodología seleccionada, pues su importancia versa en la observación in situ de los fenómenos para su mayor comprensión.

Para este efecto, y utilizando la observación directa los incidentes, se etiquetaron los fenómenos para posteriormente ser comparados de manera constante con las categorías previamente definidas, lo que permitió una nueva selección o creación de nuevas categorías.

Para la realización del presente modelo se tomó en cuenta lo siguiente:

1) Revisión de la literatura.

2) Definición del problema.

3) Delineación de los instrumentos de recolección de datos.

4) Determinación del muestreo. La población total fue de 38 universidades públicas con el modelo de educación por competencias en México.

5) Recolección de datos mediante observación directa y 50 entrevistas semiestructuradas aplicadas en las instituciones de estudio.

6) Finalmente, a medida que se desarrolló el modelo, se encontraron razones de por qué las herramientas de gestión de capital humano docente bajo el enfoque de competencias tienen efecto sobre la implementación de dicho modelo en las universidades públicas. De esta manera, se está en posibilidades de plantear un modelo objeto de la presente investigación.

Se debe acotar que no se manipularon las variables. La información se obtuvo a partir de 50 entrevistas en 38 instituciones bajo el principio de saturación teórica. La observación 

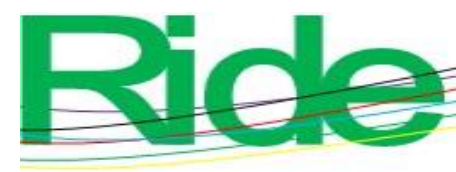

Revista Iberoamericana para la Investigación y el Desarrollo Educativo

in situ permitió que se estudiaran tal cual se han suscitado. Además, la recolección de entrevistas a través de la grabación en dispositivos electrónicos en un solo momento permitió que la investigación fuera sistemática, empírica y transversal. Toda la información obtenida fue vertida para su interpretación en el programa informático Atlas ti, ya que es un programa que facilita la creación de variables, categorías, mapas conceptuales y la compactación constante de variables.

\section{Resultados}

El modelo de gestión de capital humano docente por competencias se divide en tres etapas fundamentales:

1) Reclutamiento y selección docente por competencias, que incluye: a) la identificación de los elementos previos clave para la puesta en marcha del modelo, b) los cambios previos para la puesta en marcha del modelo y c) la forma de acercar a los candidatos a puestos docentes, así como de designar a las personas que serán los reclutadores.

2) Contratación. Que al dar curso legal a las contrataciones se dé certeza jurídica a la gestión de capital humano docente.

3) Desarrollo. En esta etapa se propone una serie de conceptos que se perfilan a una formación más que a una capacitación.

Es importante mencionar que el modelo propuesto se encuentra constituido por cuatro fases que, de manera clásica, se han retomado en la administración de recursos humanos (reclutamiento, selección, contratación y desarrollo), sin embargo, estas etapas se retoman desde una óptica de las competencias para docentes, es decir, desde antes de iniciar el proceso se recomienda hacer cambios organizacionales para la puesta en marcha del modelo, así como se hace una propuesta en competencias.

\section{Etapa I: reclutamiento y selección docente por competencias}

A continuación, se presentan los elementos previos clave para la puesta en marcha del modelo. 


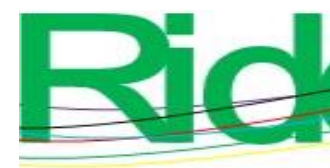

Revista Iberoamericana para la Investigación y el Desarrollo Educativo ISSN $2007-7467$

\section{Compromiso directivo}

Como parte importante en la gestión de capital humano docente, se requiere que el personal directivo y sobre todo el departamento encargado de recursos humanos se encuentre convencido de la implementación del modelo. Que sean los primeros en aplicar en su quehacer administrativo los principios, formatos y directrices de las competencias.

\section{Sensibilización}

El conocimiento de las ventajas que puede traer la aplicación de las competencias en la gestión de capital humano docente para lograr las competencias en el aprendizaje en el salón de clases es muy importante, sobre todo si se tiene conciencia de que la personalidad y las competencias del docente reclutado pueden ayudar o perjudicar en el logro de los objetivos de la institución educativa que cuenta con el modelo de competencias. Buscar la formación más que la capacitación es un factor que se debe fomentar en los involucrados, tanto directivos como personal de apoyo a la docencia y docentes mismos. En este sentido, puede recomendarse, además de la capacitación que ofrece las autoridades educativas federales, un taller vivencial donde se puedan expresar las competencias logradas por las personas a lo largo de la vida, así como las competencias potenciales que el individuo puede desarrollar y que probablemente no tengan mucho que ver con su formación académica. Lo anterior con el objeto de que el sujeto clarifique el concepto tanto de competencias docentes como no docentes. Esto puede complementarse (en la formación) con una demostración donde el docente puede mostrar sus conocimientos, habilidades, destrezas y valores, que serán evaluadas por los mismos integrantes del taller con los instrumentos que suelen utilizarse para este efecto (lista de cotejo, guía de observación, portafolio de evidencias).

\section{Identificación de las competencias institucionales rectoras}

La misión, la visión, los objetivos y filosofía de cada universidad, el decreto de creación de cada institución, la filosofía gubernamental estatal y de cada universidad, así como el plan de desarrollo institucional serán documentos básicos para determinar las competencias rectoras. Dicho trabajo puede llevarse a cabo por la alta dirección en conjunto con los directivos y los docentes de tiempo completo y parcial al momento de realizar el plan de desarrollo institucional. Lo anterior con el objeto de contar con una directriz a seguir en términos de competencias y poder trasladar dicho trabajo a todos los niveles de la institución. 


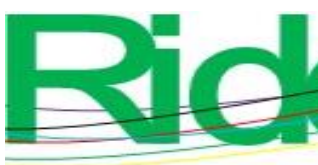

Revista Iberoamericana para la Investigación y el Desarrollo Educativo ISSN 2007 - 7467

\section{Conformación y formación continua del panel de expertos para una selección por competencias}

Una vez clarificado el concepto de competencias y determinadas las competencias institucionales rectoras, se deberá conformar el conjunto de personas que llevarán a cabo la selección de docentes en la institución. Aquí se sugiere que esté conformado por los siguientes perfiles: 1) un directivo académico, 2) un docente de área básica, 3) un docente (tiempo completo o parcial) de la especialidad a evaluar en la selección, 4) un diseñador curricular de la misma especialidad y 5) un representante de recursos humanos.

Es muy probable que solo exista uno o dos paneles de expertos en cada institución y que solo lleguen a moverse los especialistas; no se trata de conformar tantos paneles de expertos como asignaturas existan.

El trabajo de selección de los docentes de la especialidad y el diseñador curricular será de suma importancia. Con base en las competencias clave del manual de la asignatura a evaluar, llevarán a cabo el proceso de elaboración de los instrumentos de evaluación del docente aspirante, que pueden ser listas de cotejo, guías de observación o portafolio de evidencias en la especialidad de la asignatura. El trabajo del docente de área básica será evaluar esas mismas competencias en el postulante. Por su parte, el especialista en recursos humanos se encargará de evaluar los aspectos legales y de personalidad del sujeto. El directivo evaluará las competencias ejecutivas del individuo. Y finalmente, el panel de expertos deberá enfocarse en una actualización constante sobre los avances que la sociedad del conocimiento genere en términos de competencias y en tecnología educativa con el fin de modificar los instrumentos de selección conforme dichos avances. Paralelamente, se presentan los cambios previos para la puesta en marcha del modelo.

\section{Propuesta de mejora al manual de organización de la institución bajo el modelo educativo por competencias}

El documento administrativo que proporciona toda la información acerca de las jerarquías, funciones y obligaciones de todo el personal de la organización es el manual de organización. Cabe mencionar que sería idónea la modificación de todo el documento de acuerdo con los requerimientos del modelo educativo por competencias. Sin embargo, el presente modelo solo sugiere el cambio concerniente a la gestión de capital humano docente 

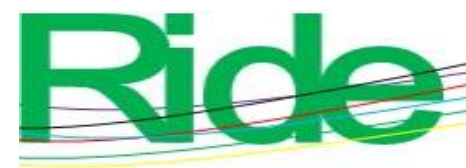

Revista Iberoamericana para la Investigación y el Desarrollo Educativo ISSN 2007 - 7467

y que este cambio sea revisado por el panel de expertos con el fin de dar cumplimiento a las expectativas del modelo educativo.

\section{Determinación de las competencias genéricas y competencias específicas en el manual de organización para la selección de capital humano}

El panel de expertos revisará las funciones de cada integrante del departamento de recursos humanos. Posteriormente, se enfocará en la determinación de las competencias de cada uno de los puestos del departamento de recursos humanos tanto genéricas como específicas.

\section{Determinación de las competencias genéricas y específicas docentes}

El panel de expertos deberá proponer al departamento de planeación las competencias genéricas que deberán poseer los docentes para ser contratados y estas deberán ser plasmadas en el manual de organización con el afán de que en los puestos docentes se tengan identificadas las competencias para futuras contrataciones.

Por parte de las competencias específicas, las determina el docente experto en la materia y el diseñador curricular de la carrera. Serán tomadas en cuenta en la evaluación del candidato por el panel de expertos al momento de la clase muestra y se plasmarán en una lista de cotejo o guía de observación que diseñará el panel para este efecto o portafolio de evidencias.

\section{Propuesta de mejora de los procedimientos de calidad referentes a la gestión de capital humano docente alineados a competencias}

Los procedimientos de gestión de la calidad tienen la virtud de ser susceptibles de ser modificados a través de lo que se conoce como mejora continua. En este sentido, es clave que los procedimientos concernientes a la gestión de capital humano docente sean revisados por el panel de expertos para que cumplan no solo con los requerimientos de la misma norma, sino, además, los requerimientos de competencias y las necesidades académicas de la institución. 


\section{Propuesta de mejora a la reglamentación de ingreso, promoción y permanencia}

La propuesta de mejora a esta reglamentación se enfoca en los siguientes puntos:

1) Manejar competencias del docente en los niveles establecidos para determinar los tabuladores.

2) Verificar que en el examen de oposición se consideren a las competencias como complemento de la evaluación.

3) Que las necesidades de personal de las áreas académicas que se envían al departamento de recursos humanos incluyan las competencias tanto genéricas como específicas requeridas por el puesto para su mejor identificación.

4) Que en la convocatoria pública para solicitar personal docente se anote (además de los requisitos que marca el reglamento) las competencias requeridas por el manual de asignatura y por el manual de organización.

5) Que las evaluaciones de ingreso las realice el panel de expertos con base en competencias.

6) Que en la evaluación para promoción se considere, además de los puntos que marca la reglamentación de ingreso, promoción y permanencia, el desarrollo de competencias tanto docentes como no docentes.

En cuanto al reclutamiento y selección por competencias se muestran las consideraciones siguientes:

- $\quad$ El departamento de recursos humanos emitirá una convocatoria para las vacantes docentes tratando de reunir, más que los perfiles, las competencias que se requieren. Lo anterior con base en las competencias genéricas y específicas requeridas y que ya se encuentran determinadas en el manual de organización. Se trata de que al candidato se le solicite un portafolio de evidencias, además de su currículo, y sea presentado en su clase muestra. También se deberán requerir en la convocatoria las competencias no docentes desarrolladas en la empresa o empleos anteriores.

- Una vez habiendo recibido las solicitudes, descartar aquellas que no cumplan con las competencias requeridas y citar a entrevista a los candidatos idóneos (presencial o virtual). Dicha entrevista será en tres partes: una con el encargado de recursos humanos, posteriormente con el especialista en la materia a impartir y, por último, con el director o coordinador del área académica. Se descartará a aquellos que no reúnan las competencias en la entrevista. Y se solicitará a los candidatos más competentes que se presenten a una clase 


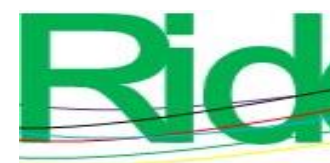

\section{Revista Iberoamericana para la Investigación y el Desarrollo Educativo ISSN 2007 - 7467}

muestra (presencial o virtual), donde se evaluarán las competencias genéricas, específicas, docentes y no docentes. Una vez terminado y seleccionado al candidato idóneo en función de sus competencias, se turnarán a recursos humanos los instrumentos de evaluación con su dictamen, que puede ser: $a$ ) competente, $b$ ) básico, $c$ ) umbral, $d$ ) no competente. A partir de ello se llevará a cabo o no su contratación, sin perder de vista la aplicación de pruebas psicométricas que permitan identificar en el candidato la personalidad, las habilidades, destrezas, valores y competencias que posea o que potencialmente pueda desarrollar el candidato. Todo lo anteriormente descrito se puede ver gráficamente en la figura 2.

Figura 2. Etapa I: reclutamiento y selección de capital humano docente por competencias

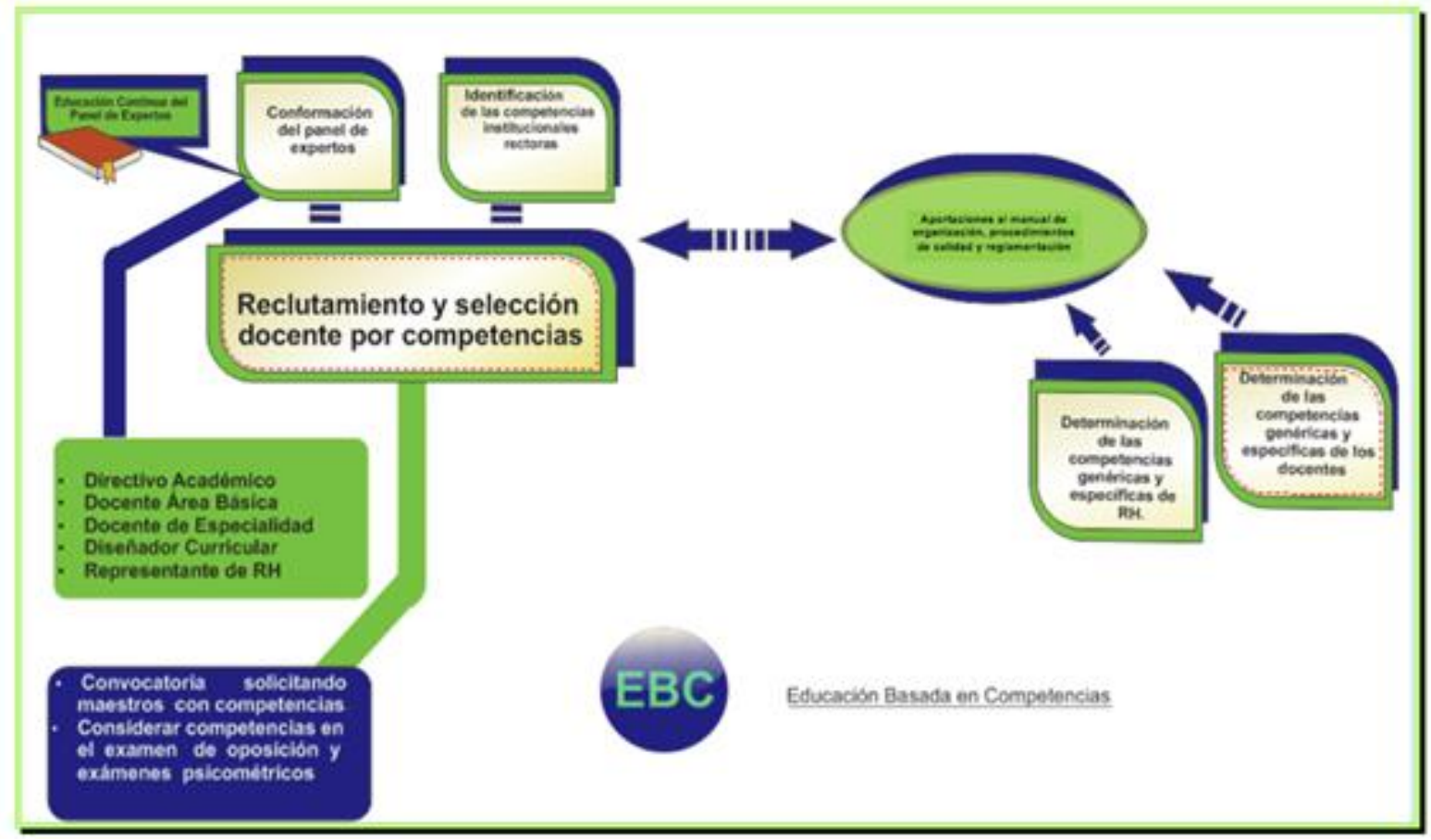

Fuente: Elaboración propia con datos de la investigación

\section{Etapa II: contratación de capital humano por competencia}

En el capítulo III de la Ley Federal del Trabajo en México ("De la productividad, formación y capacitación de los trabajadores”), en los artículos 153 A, C, D, H, U y V, el término competencia laboral, lo cual resulta un respaldo legal al objeto de existencia del presente modelo. Son también recurrentes en la misma ley términos tales como conocimientos, habilidades y destrezas. En efecto, la Ley Federal del Trabajo en México considera de manera intrínseca el concepto de competencias. A su vez, la Ley General de 

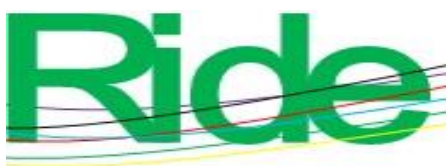

Revista Iberoamericana para la Investigación y el Desarrollo Educativo

ISSN 2007 - 7467

Educación en México, en el artículo 4,5 establece: "La formación para el trabajo procurará la adquisición de conocimientos, habilidades o destrezas, que permitan a quien la recibe desarrollar una actividad productiva demandada en el mercado, mediante alguna ocupación o algún oficio calificados ”.

La contratación de personal docente por competencias (propuesta en este modelo) no difiere de la figura legal plasmada en la Ley Federal del Trabajo, dado que considera el concepto de competencias como elemento de desempeño del trabajo o de terminación de la relación laboral, según el artículo 47 fracción I, que establece que es causa de rescisión de la relación de trabajo, sin responsabilidad para el patrón:

Engañarlo el trabajador o en su caso, el sindicato que lo hubiese contratado con certificados falsos o referencias en los que le atribuyan al trabajador la capacidad, aptitudes o facultades de que carezca. Esta causa de rescisión dejará de tener efecto después de los treinta días de prestar sus servicios el trabajador.

En este sentido, se puede interpretar que los certificados o referencias a los que alude el artículo anterior pueden ser certificaciones laborales o evidencias que presente el trabajador al momento de su contratación. La etapa de contratación de capital humano por competencias se puede ver en la figura 3 .

Figura 3. Etapa II: contratación de personal docente por competencias

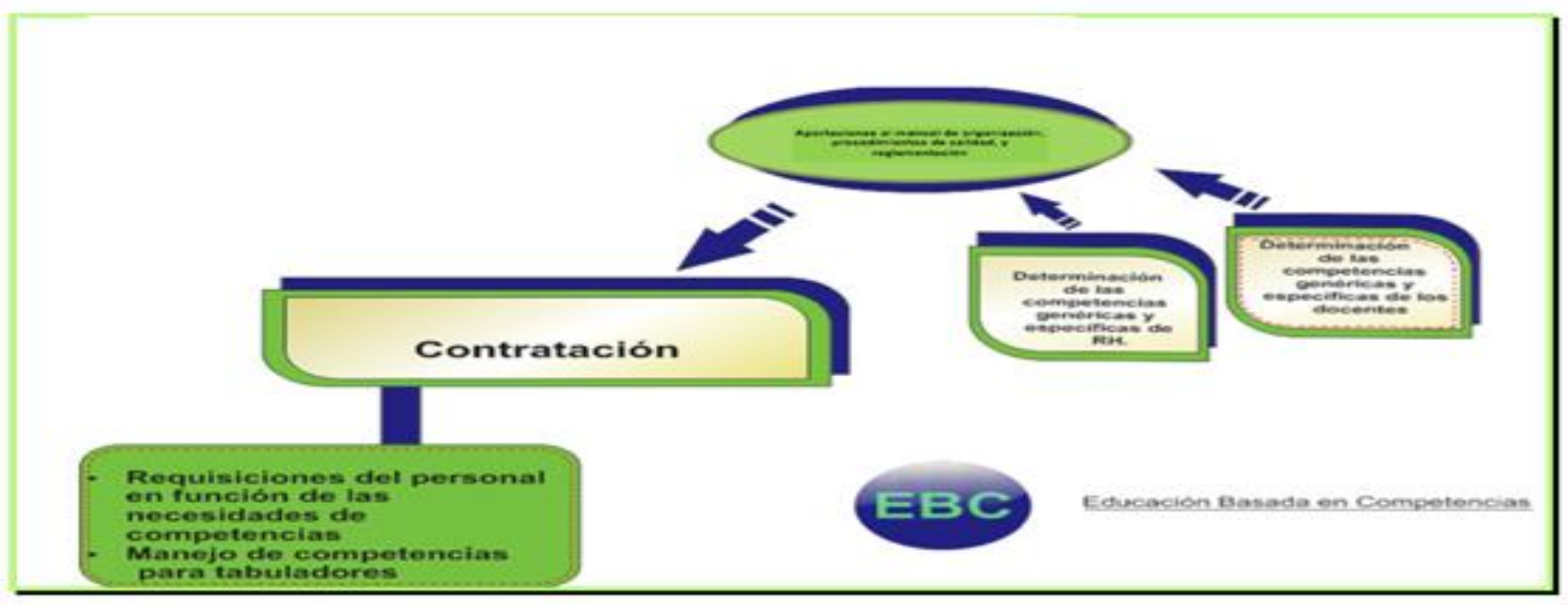

Fuente: Elaboración propia 


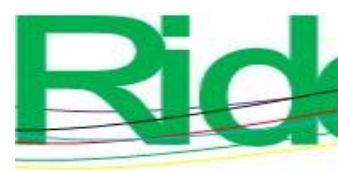

Revista Iberoamericana para la Investigación y el Desarrollo Educativo ISSN 2007-7467

\section{Etapa III: desarrollo de capital humano docente por competencias}

La propuesta de este modelo busca ir más allá de un desarrollo docente a través de la capacitación, es decir, busca una formación constante, considerando los siguientes elementos.

\section{Inducción adecuada del docente}

Una vez llevada a cabo la contratación con base en las competencias más que en el perfil, se trata de situar al docente contratado, así como dar a conocer los lugares clave en el desarrollo de su labor docente, es importante que el docente conozca el modelo EBC, impartir a las nuevas contrataciones un curso-taller formativo que dé inicio de actividades puedan identificar sus competencias tanto genéricas como específicas además de las competencias que por formación el sujeto posea pero así como las competencias que potencialmente pueda desarrollar y que quizá no haya descubierto, se propone, además, tal como el modelo EBC determina tutores para alumnos, exista una tutoría a los nuevos profesores en el manejo de los formatos e instrumentos de evaluación que sean requeridos por el modelo, dicha tutoría puede servir para resolver dudas de los recién contratados en la aplicación y desarrollo de competencias en el salón de clases, evaluaciones, asesorías, tutoría a alumnos.

\section{Formación permanente en la EBC}

Se debe considerar que la mayor parte de la formación de los docentes ha sido "tradicional" y que el modelo por competencia establece una distribución de $70 \%$ práctico y $30 \%$ teórico (en la mayoría de las universidades públicas en México). En este sentido, y pensando que los docentes deben permitir el acopio de las competencias a los alumnos, se tiene que concebir una formación docente continua, y no una capacitación temporal, tomando en cuenta los siguientes puntos:

1) Establecer cursos de capacitación con base en las necesidades docentes y el desarrollo de competencias de los integrantes de un grupo de docentes con carreras afines.

2) Seguimiento permanente de la aplicación del modelo por competencias en el aula física o virtual.

3) Seguimiento de la aplicación plena de los manuales de asignatura y de los instrumentos de evaluación sugeridos en el mismo. 

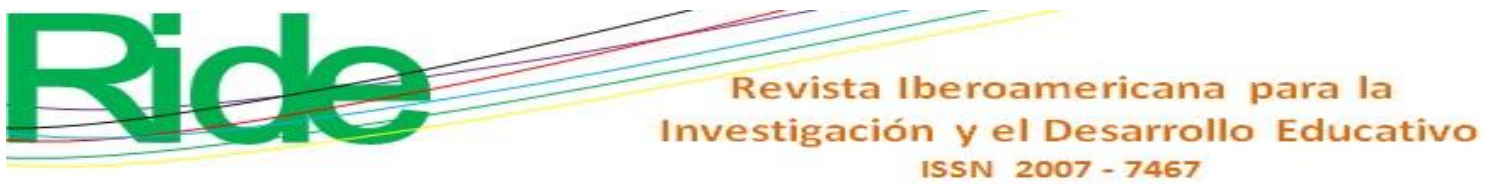

4) Capacitación y entrenamiento in situ, es decir, obtener la vinculación del sector productivo para lograr estancias del personal docente en su área de conocimiento con el objeto de involucrar al mismo personal en la realidad empresarial de su entorno. Así, al término de dicha estancia, el docente habrá desarrollado competencias no docentes que permitirán cumplir con el modelo por competencia (30 \% teórico y $70 \%$ práctico).

5) Seguimiento de la formación proporcionada al docente.

Todo lo anteriormente descrito se puede apreciar gráficamente en la figura 4.

Figura 4. Etapa III: desarrollo de personal docente por competencias

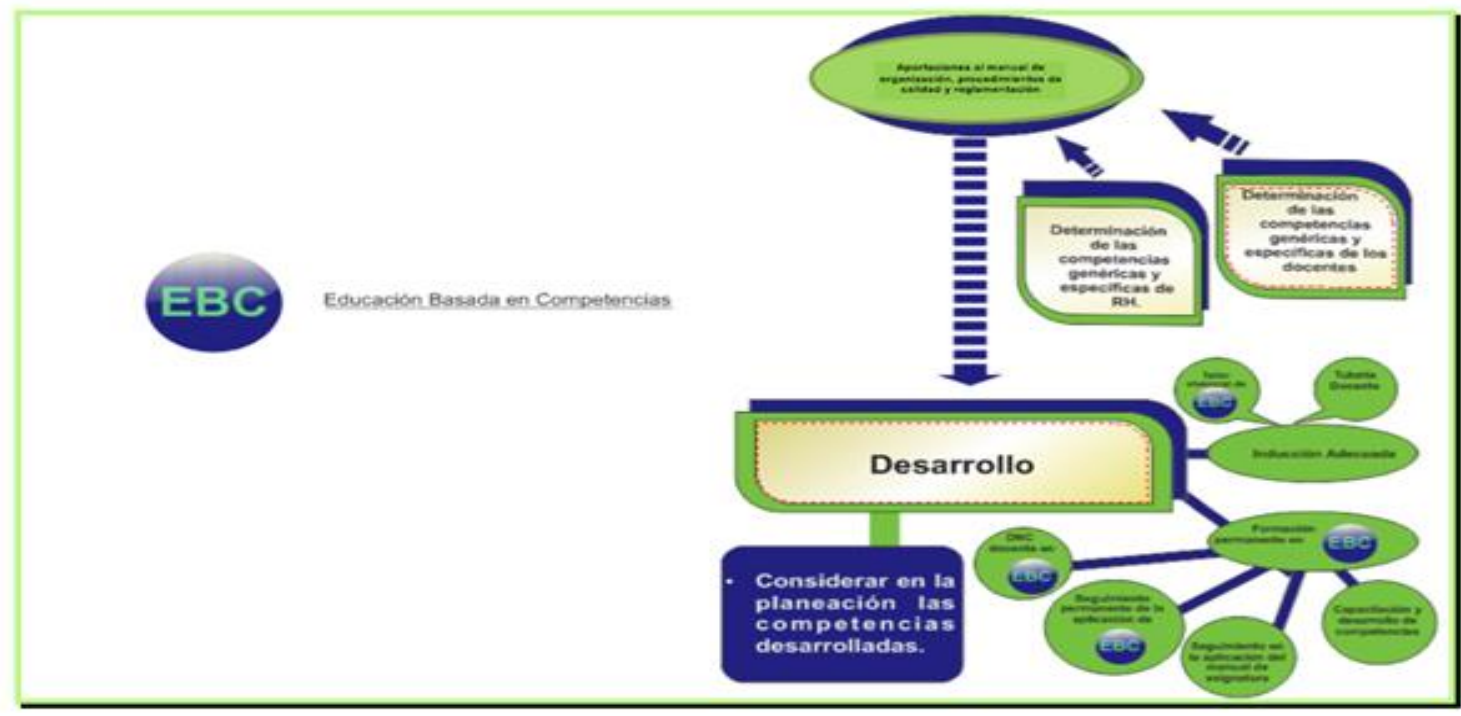

Fuente: Elaboración propia

En la figura 5, por su parte, se presenta el modelo de gestión de capital humano por competencias de forma holística, integrando todas y cada una de sus partes. 

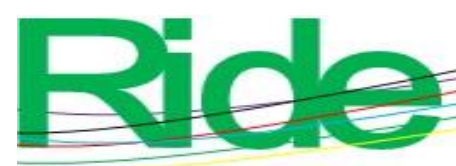

Revista Iberoamericana para la

Investigación y el Desarrollo Educativo

ISSN $2007-7467$

Figura 5. Modelo de gestión de personal docente por competencias

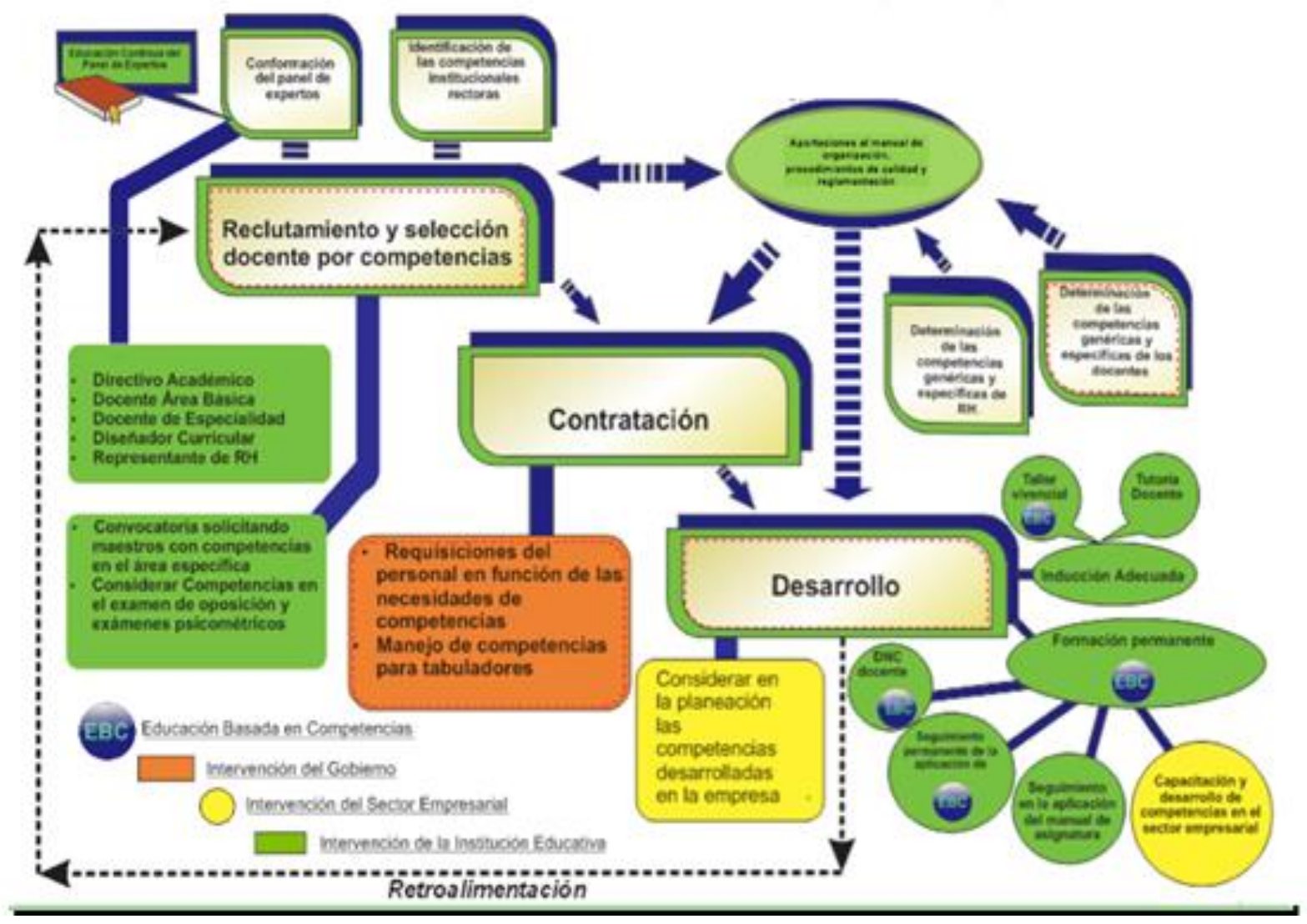

Fuente: Elaboración propia

\section{Relación de los resultados obtenidos con la teoría fundamentada}

Los beneficios derivados del uso de la teoría fundamentada en la construcción del modelo de gestión de capital humano antes descrito versan en una incursión de estudios de gestión organizacional donde se busca destacar la importancia que tiene establecer una estructura que permita dejar ver elementos administrativos y de gestión con base en fuentes primarias y sus actores sociales en su vida y quehacer diario en sus organizaciones. Se consideró la teoría fundamentada en la gestión de capital humano como herramienta metodológica y de análisis de información empírica, lo que permitió la obtención de datos provechosos para la construcción de un modelo que sirva para la construcción de nuevo conocimiento.

En este sentido, es importante enfatizar en que la teoría fundamentada como herramienta metodológica permite la generación de herramientas de gestión en las instituciones educativas. Desde Glaser y Strauss (2017) y su teoría fundamentada hasta la 


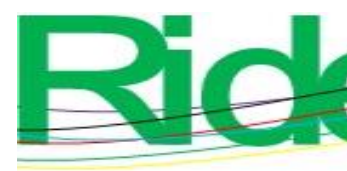

Revista Iberoamericana para la
Investigación y el Desarrollo Educativo ISSN 2007-7467

concepción de Raymond (2005), la construcción del conocimiento aporta elementos para incursionar en el área de administración y gestión con el fin de reconocer la aplicabilidad en esta rama del saber y dejar evidencia de la utilidad que tiene la investigación cualitativa, y en específico la teoría fundamentada en la gestión de capital humano.

\section{Discusión}

Es importante mencionar que el modelo tiene como entrada el reclutamiento y selección y como salida el desarrollo. El modelo de gestión de personal docente por competencias es cíclico, pero no cerrado, pues está abierto a la retroalimentación que el desarrollo mismo puede dar a las nuevas formas de reclutamiento; es actualizable en función de los avances que la gestión de capital humano tenga, así como en función de la participación de la parte gubernamental, del sector empresarial y de la misma institución educativa, y es holístico porque considera elementos tanto internos como externos del entorno. También es igualmente importante advertir que algunos elementos intervienen en más de una fase, tal es el caso de las aportaciones al manual de organización y a los procedimientos de calidad, y los elementos que derivan de ahí, lo anterior con fundamento en que el manual de organización es un documento rector en cualquier organización.

Ahora bien, en un ejercicio comparativo con algunos de los estudios ya citados anteriormente, Alles (2017) identifica 20 elementos en su modelo de gestión por competencias, desde una gestión por competencias hasta la incorporación y promociones internas del personal, lo cual contribuye a una selección efectiva de personal por competencias. Es de valorar la incursión de las competencias en las entrevistas, punto de convergencia entre ambas propuestas, sin embargo, es también importante hacer notar que no está dirigido al personal docente y mucho menos a personal de universidades públicas, que es la aportación a la gestión del conocimiento del presente trabajo.

En esa misma línea, Hellriegel (2009) combina las competencias centrales con los principios administrativos y basa su conocimiento en la autoadministración, acción estratégica, multicultural, trabajo en equipo, planeación, administración y comunicación, y como punto de convergencia señala a la aplicación de las competencias en una gestión integral del conocimiento en la organización; sin embargo, no se encuentra un punto de coincidencia para el sector docente. 


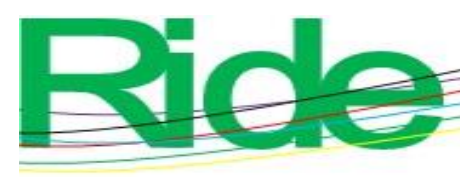

Revista Iberoamericana para la Investigación y el Desarrollo Educativo ISSN $2007-7467$

\section{Conclusiones}

El modelo de gestión de capital humano por competencias para universidades públicas en México presentado tiene como columna vertebral los conceptos de reclutamiento, selección, contratación y desarrollo, y una propuesta descrita de la aplicación en la gestión de personal docente en universidades públicas. Al integrar en lo posible todos los elementos de manera holística en los procesos de gestión, se logra el objetivo planteado en la presente investigación: generar una herramienta de aplicabilidad inmediata para las universidades cuyo modelo educativo está basado en la promoción de competencias y que, sin embargo, su gestión de personal docente no considera las mismas en su actuar diario.

Lo anterior permite identificar que, como todo modelo, puede ser perfectible, y si bien se están considerando elementos de la actual forma de trabajo de manera híbrida en las universidades públicas del país, se puede ir actualizando conforme se vaya modificando la forma en que vayan evolucionando las actividades.

Las propuestas que se derivan del modelo presentado son que todos los actores involucrados en la gestión de personal docente logren participar de manera activa, según lo descrito, para lograr un cambio en el paradigma de la contratación de docentes, que en ocasiones se realiza solo por el conocimiento de su perfil profesional, el cual no siempre empata con las competencias desarrolladas en su actuar profesional y personal.

Por otra parte, es pertinente que desde el proceso de reclutamiento en las convocatorias emitidas se incluyan las competencias requeridas para el puesto docente a cubrir; encaminar así al candidato a una asignatura que quizá no sea de su perfil profesional pero que, sin embargo, tenga las competencias y certificaciones para impartirlas: ampliar su abanico de posibilidades laborales.

Es importante hacer notar que el docente debe cumplir con el requerimiento de la educación por competencias: 30 por ciento teórico, 70 por ciento práctico. La crítica más frecuente a la integración práctica del docente en empresas es obvia: “¿Cómo saber que el docente que envías a la empresa va a regresar a la docencia?”. La respuesta la ha dado la historia: aquellas personas que tienen la vocación docente siempre serán docentes, y esa es la competencia que, para los que se encuentran frente a un grupo en un salón de clases presencial o virtual, se debe descubrir, fomentar y desarrollar. 


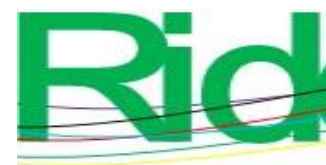

Revista Iberoamericana para la
Investigación y el Desarrollo Educativo
ISSN $2007-7467$

Futuras líneas de investigación

Las siguientes líneas de investigación se pueden extraer del modelo aquí diseñado:

- $\quad$ Análisis comparativo con diferentes subsistemas para su adaptación en ellos.

- Integración de estudiantes al proceso de gestión de capital humano docente siendo ellos los participantes activos en el proceso de aprendizaje.

- Reclutamiento: es de interés estudiar las nuevas formas de reclutamiento electrónico, las cuales incluyen en el candidato competencias en el uso de tecnologías de información.

- Selección: es quizá uno de los puntos con mayor peso en la gestión de capital humano, sobre todo en el estudio de las entrevistas, donde se descubran las competencias de los individuos; y sin duda alguna causa interés el estudio de los exámenes psicométricos diseñados por competencias tomando como base la teoría escrita para moldear instrumentos psicométricos que no solo describan las competencias del candidato, sino que presente nuevas competencias que el individuo posea y que, quizá, no haya descubierto. Lo anterior para mejorar la gama laboral del docente.

- Desarrollo: es de especial atención para estudios posteriores dos conceptos que se han acuñado y que como aportación a la gestión del conocimiento en el presente modelo se describen: 1) tutoría docente, acompañamiento del nuevo docente durante los primeros meses por parte de otro docente que conozca el modelo por competencias con el fin de guiar la aplicación correcta de este hasta saber que ya domina no solo el manejo de formatos, instrumentos de evaluación y manuales, sino también el modelo mismo; 2) competencias no docentes, aquellas competencias que el docente debe desarrollar en estancias en la empresa (siendo docente) bajo una modalidad contractual bien definida en la institución educativa. 


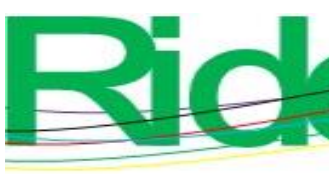

Revista Iberoamericana para la Investigación y el Desarrollo Educativo ISSN $2007-7467$

\section{Referencias}

Acevedo, J. A., García, A., Aragón, M. del M. y Oliva, J. M. (2017). Modelos científicos: significado y papel en la práctica científica. Revista Científica, 30(3), 155-166. Recuperado de https://doi.org/10.14483/23448350.12288.

Adúriz, B, A. e Izquierdo, M. (2009). Un modelo de modelo científico para la enseñanza de las ciencias naturales. Revista Electrónica de Investigación en Educación en Ciencias, 4(1), 40-49. Recuperado de https://www.redalyc.org/articulo.oa?id=273320452005.

Alles, M. (2017). Selección por competencias. México: Granica.

Alles, M. (2018). Desempeño por competencias. México: Granica.

Bailer, D. (2013). Scientific Models in Philosophy of Science. Pittsburgh, United States: University of Pittsburgh Press. https://upittpress.org/books/9780822962731/

Chiavenato, I. (2018). Administración de recursos humanos. El capital humano en las organizaciones. México: McGraw-Hill.

Chiavenato, I. (2020). Gestión del talento humano. Chile: McGraw-Hill

Cuesta, A. (2010). Tecnología de gestión de recursos humanos (3. a ed.). La Habana, Cuba: Félix Varela y Academia.

Cuesta, A. y Valencia, M. (2018). Capital humano: contexto de su gestión. Desafíos para Cuba. Ingeniería Industrial, 39(2), 135-145. Recuperado de http://scielo.sld.cu/scielo.php?script=sci_arttext\&pid=S1815$59362018000200135 \& \operatorname{lng}=$ pt\&tlng=es.

Giere, R. (2004). How Models Are Used to Represent Reality. Philosophy of Science, 71(5), 742-752. Retrieved from https://www.jstor.org/stable/10.1086/425063.

Glaser, B. and Strauss, A. (2017). The Discovery of Grounded Theory. Strategies for Qualitative Research. United States: Routledge Taylor \& Francis Group.

Gómez, C. y Mendoza, L. (2013). Modelo de gestión por competencias para la empresa Acmed S. A.S. (Proyecto de grado). Universidad de Cartagena, Cartagena. Recuperado de

https://repositorio.unicartagena.edu.co/bitstream/handle/11227/445/TESIS\%20DE\% 20GRADO\%20\%20MODELO\%20DE\%20GESTI\%c3\%93N\%20POR\%20COMPE TENCIAS\%20PARA\%20LA\%20.pdf? sequence=1\&isAllowed=y. 

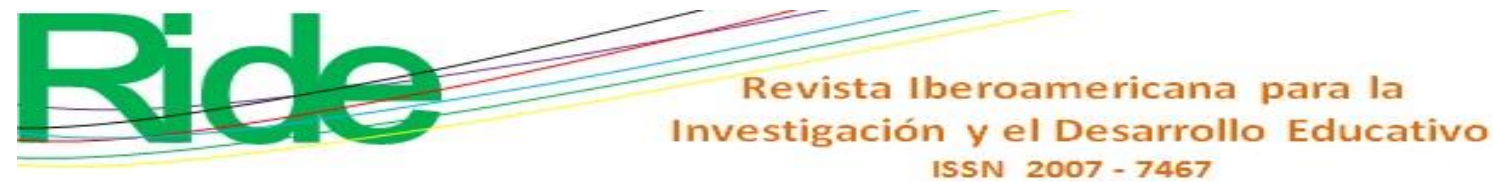

Gómez, N. (2010). La teoría fundamentada y el estudio de casos. Miríada, 3(5), 1-27. Recuperado de http://p3.usal.edu.ar/index.php/miriada/article/view/17/169.

Hellriegel, D. (2009). Administración: un enfoque basado en competencias. México: Cengage Learning Editores.

Hubert, B. (2019). La teoría fundamentada como método de investigación cualitativa para explicar la ausencia del estado en los diferentes niveles de emprendimientos. Recimundo: Revista Científica de la Investigación y el Conocimiento, 3(1), 742-761. Recuperado de https://dialnet.unirioja.es/servlet/articulo?codigo=6796770.

Raymond, E. (2005). La teorización anclada (grounded theory) como método de investigación en ciencias sociales: en la encrucijada de dos paradigmas. Cinta de Moebio, (23). Recuperado de https://1library.co/document/8ydpx2gy-teoriafundamentada-raymond-pdf.html.

Rivero, A., Dabos, G., Marino, J. y Rodríguez, M. C. (2014). El método de teoría fundada: desarrollo de una aplicación práctica para analizar el impacto de la formación gerencial de postgrado en las transiciones de carrera de sus graduados. Recuperado de https://www.researchgate.net/publication/310477107_El_metodo_de_teoria_fundad a_Desarrollo_de_una_aplicacion_practica_para_analizar_el_impacto_de_la_formac ion_gerencial_de_postgrado_en_las_transiciones_de_carrera_de_sus_graduados.

Rodríguez, O. (2015). Diseño del sistema de gestión integral de capital humano (SGICH) en la empresa constructora militar "El Vaquerito". (Tesis de maestría). Universidad Central Marta Abreu de las Villas, Santa Clara. Recuperado de https://dspace.uclv.edu.cu/handle/123456789/6281?show=full.

Restrepo, L. S., Landino, A. M. y Orozco, D. C. (2008). Modelo de reclutamiento y selección de talento humano por competencias para niveles directivos de la organización. Scientia et Technica. 14(39), 286-291. Recuperado de https://www.redalyc.org/pdf/849/84920503051.pdf.

Reyes, A. (2012). Administración de personal, Relaciones humanas. México: Limusa Noriega.

Saracho, J. M. (2005). Un modelo general de gestión por competencias. Santiago, Chile: RIL editores. 

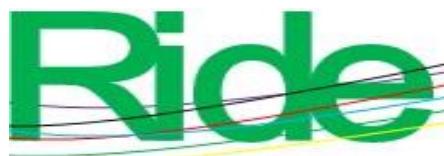

Suárez, M., (2010). Scientific Representation. Philosophy Compass, 5(1), 91-101. Retrieved from https://doi.org/10.1111/j.1747-9991.2009.00261.x.

Tejada, A. (2003). Los modelos actuales de gestión en las organizaciones: gestión del talento, gestión del conocimiento y gestión por competencias. Psicología desde el Caribe, (12), 115-133. 


\begin{tabular}{|c|c|}
\hline Rol de Contribución & Autor (es) \\
\hline Conceptualización & $\begin{array}{l}\text { Raymundo Lozano Rosales (igual) } \\
\text { María de Lourdes Amador Martínez (igual) }\end{array}$ \\
\hline Metodología & $\begin{array}{l}\text { Raymundo Lozano Rosales (igual) } \\
\text { María de Lourdes Amador Martínez (igual) }\end{array}$ \\
\hline Software & $\begin{array}{l}\text { Raymundo Lozano Rosales (principal) } \\
\text { María de Lourdes Amador Martínez (que apoya) }\end{array}$ \\
\hline Validación & $\begin{array}{l}\text { Raymundo Lozano Rosales (principal) María de Lourdes } \\
\text { Amador Martínez (igual) Mariza Raluy Herrero (igual) }\end{array}$ \\
\hline Análisis Formal & $\begin{array}{l}\text { Raymundo Lozano Rosales (principal) María de Lourdes } \\
\text { Amador Martínez (igual) Mariza Raluy Herrero (igual) }\end{array}$ \\
\hline Investigación & $\begin{array}{l}\text { Raymundo Lozano Rosales (igual) } \\
\text { María de Lourdes Amador Martínez (igual) }\end{array}$ \\
\hline Recursos & $\begin{array}{l}\text { Raymundo Lozano Rosales (igual) } \\
\text { María de Lourdes Amador Martínez (igual) } \\
\text { Mariza Raluy Herrero (igual) }\end{array}$ \\
\hline Curación de datos & $\begin{array}{l}\text { Raymundo Lozano Rosales (principal) } \\
\text { María de Lourdes Amador Martínez (igual) } \\
\text { Mariza Raluy Herrero (igual) }\end{array}$ \\
\hline $\begin{array}{l}\text { Escritura - Preparación del } \\
\text { borrador original }\end{array}$ & $\begin{array}{l}\text { Raymundo Lozano Rosales (Igual) } \\
\text { María de Lourdes Amador Martínez (principal) } \\
\text { Mariza Raluy Herrero (igual) }\end{array}$ \\
\hline $\begin{array}{l}\text { Escritura - Revisión y } \\
\text { edición }\end{array}$ & $\begin{array}{l}\text { Raymundo Lozano Rosales (Igual) } \\
\text { María de Lourdes Amador Martínez (principal) } \\
\text { Mariza Raluy Herrero (igual) }\end{array}$ \\
\hline Visualización & $\begin{array}{l}\text { Raymundo Lozano Rosales (principal) } \\
\text { María de Lourdes Amador Martínez (igual) } \\
\text { Mariza Raluy Herrero (igual) }\end{array}$ \\
\hline Supervisión & Raymundo Lozano Rosales (principal) \\
\hline Administración de Proyectos & $\begin{array}{l}\text { Raymundo Lozano Rosales (principal) } \\
\text { María de Lourdes Amador Martínez (igual) } \\
\text { Mariza Raluy Herrero (igual) }\end{array}$ \\
\hline Adquisición de fondos & $\begin{array}{l}\text { Raymundo Lozano Rosales (principal) } \\
\text { María de Lourdes Amador Martínez (que apoya) } \\
\text { Mariza Raluy Herrero (que apoya) }\end{array}$ \\
\hline
\end{tabular}

